\title{
MIS Distal Metatarsal Metaphyseal Osteotomy in the treatment of metatarsalgia: MOXFQ patient reported outcomes
}

\author{
W.McMurrich, A. Peters, M. Ellis, G.Baer, D. MacDonald, H. Shalaby, JC. McKinley
}

\begin{abstract}
Introduction

The aim of this paper is to present validated patient reported outcomes for MIS Distal Metatarsal Metaphyseal Osteotomy (DMMO) in the treatment of metatarsalgia. We aim to evaluate the DMMO procedure, report patient satisfaction with the operated foot and report any complications of this procedure.
\end{abstract}

\section{Patients and Methods}

Between 2014 and 2016, patients who had failed conservative treatment for metatarsalgia were identified in the orthopaedic outpatient clinic. Twenty four consecutive patients requiring DMMO plus/minus toe straightening were prospectively studied. Patients requiring additional procedures at the time of surgery were excluded. Patients completed the validated Manchester-Oxford Foot Questionnaire (MOXFQ) three weeks pre-operatively and 1 year postoperatively. The MOXFQ results were analysed using Paired t-tests. A supplementary question was asked regarding patient satisfaction with the operated foot.

\section{Results}

There were 20 women and 4 men with a mean age of 64 years (sd 8.6). Statistically significant differences were found between the pre and 
postoperative MOXFQ. The postoperative MOXFQ score demonstrated a poorer result for two patients, no change for two patients and improvement in 20 patients, with four of these patients recording the lowest possible score. There was a 29.5 point improvement in mean metric MOXFQ Index score. Seventynine percent $(n=19)$ of patients were satisfied or very satisfied with the operated foot. The average recoil of the metatarsal heads following DMMO was M2 $4.01 \mathrm{~mm}, \mathrm{M} 34.55 \mathrm{~mm}, \mathrm{M} 44.16 \mathrm{~mm}$. There was one delayed union and no nonunions. Further reported complications were a gastric bleed, pulmonary embolism (VTE), and one intra operative broken burr.

\section{Conclusion}

Our study demonstrates a clinically important and statistically significant improvement in patient reported outcomes following DMMO, with $79 \%$ of patients satisfied or very satisfied with this procedure. The average recoil of the metatarsal heads following DMMO was M2 4.01mm, M3 4.55mm, M4 4.16mm with one delayed union and no non-unions.

Key words; Minimally invasive surgery, Forefoot Disorders, Validated patient reported outcome measure, metatarsalgia, Distal Metatarsal Metaphyseal Osteotomy, Manchester Oxford Foot Questionnaire

Level IV evidence. 


\section{Introduction}

The use of Minimally Invasive Surgery (MIS) for foot surgery is relatively new procedure and there is limited independent research available to evidence its effectiveness [1-5]. As it is a new procedure and there will be a learning curve for those who will undertake the procedure, it is important to evaluate and share results to improve practice.

The incidence of metatarsalgia is reported to be $10 \%$ in the general population $[2,6]$. Metatarsalgia is caused by excessive pressure across the metatarsal heads and is multi-factorial in origin. Excessive pressures may be caused by a number of factors including increased body weight, activity (walking or running), footwear with an increased heel height, tight Achilles tendon, weight distribution imbalance across the forefoot [7], and an inefficient first ray [6,8]. The correction of a hallux valgus deformity may reduce the pressures on the lesser metatarsals, but there are no defined parameters to guide when a hallux valgus correction alone is sufficient to treat metatarsalgia [8].

Conservative treatments of metatarsalagia include callus debridement, orthoses/padding, shoe modifications, lifestyle changes to modify the time walking or running, weight management and Achilles tendon stretching. Surgical interventions may be considered when conservative treatments fail. It is suggested that metatarsal shortening or elevation is a requirement to reestablish joint stability $[6,7,9]$.

A formula for metatarsal length has been proposed for pre-surgical planning [7]. It is proposed that the ideal formula is to have lateral sesamoid alignment with the $4^{\text {th }}$ metatarsal head. The second metatarsal should be longer than the $1^{\text {st }}$ metatarsal by $1-3$ millimetres and the $3^{\text {rd }}, 4^{\text {th }}$ and $5^{\text {th }}$ metatarsals progressively shorter than the $2^{\text {nd }}$. A metatarsal osteotomy aims to correct and improve the 
weight distribution across the metatarsals heads. Currently the standard method of correcting the metatarsal position is a Weil osteotomy [10]. However, commonly reported complications include stiffness of the joint [2], 36\% (233 of 1131), a "floating toe", $15 \%$ recurrence, $7 \%$ transfer metatarsalgia and $3 \%$ nonunion/malunion [11].

The clinical indications for DMMO include pain under/around the metatarsal heads (especially with weight bearing), presence of plantar callus, abnormal metatarsal parabola and subluxations of the metatarsophalangeal joints. DMMO aims to correct the metatarsal parabola and reduce the forefoot overloading or improve the forefoot loading. Proponents of DMMO argue that the major advantages are the very small wound and minimal stripping of soft tissues, as an extra articular procedure there is less risk of avascular necrosis, it results in less MTP joint stiffness, there are no internal fixations and hence reduced risk of infection, implant breakage or displacement [12].

DMMO is designed to restore the lesser metatarsals ' position and distribute weight bearing forces. It is proposed that for the treatment of metatarsalgia to be successful DMMO of more than one metatarsal is required [6]. The repositioning of the metatarsal heads may occur through shortening, elevation, or both (see Figure 1). Immediate weight bearing postoperatively means that the metatarsal heads find a position according to the weight-bearing pattern of the patient [2]. Metatarsal lengths are set upon weight bearing and tension of the surrounding soft tissue structures. 


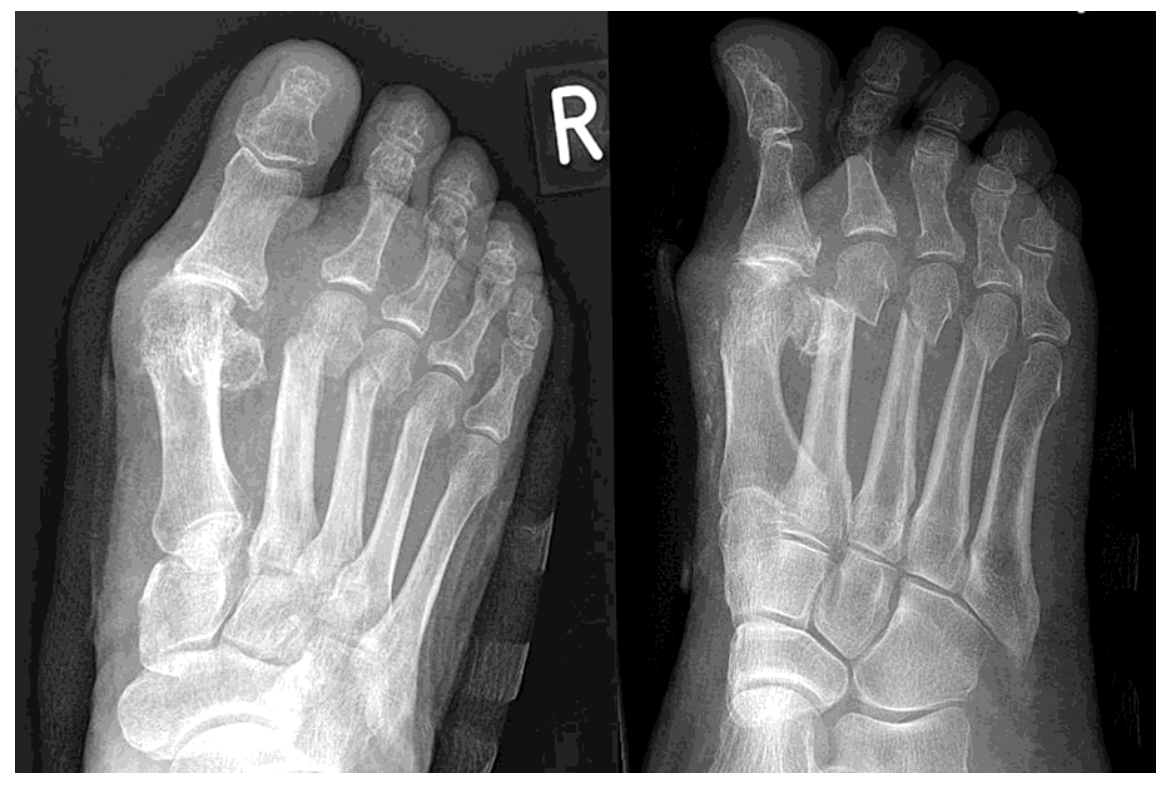

Figure 1. Postoperative X-rays following DMMO

The current study aims to evaluate the effectiveness of the procedure using patient-reported measures. We are interested to determine whether the DMMO procedure is associated with clinically and statistically significant differences in MOXFQ [13] scores pre to postoperatively.

This study aim to evaluate the effectiveness of the DMMO procedure at 1 year postoperative using MOXFQ patient reported outcome scores and satisfaction using a 5-point likert scale.

\section{Method}

The project obtained a favourable opinion from the local Research Ethics Committee, classified by the NHS Research and Development Office and registered with the University database.

\section{Participants}

Between October 2014 and February 2016 the $2^{\text {nd }}, 3^{\text {rd }}, 4^{\text {th }}$ DMMO procedure plus or minus toe straightening (flexor tenotomy, PIP joint fusion or MIS straightening) was offered to patients who had failed at least 6 months of conservative treatment for metatarsalgia. The definition of static mechanical 
metatarsalgia is clinically assessed mechanical plantar lesser metatarsal head pain when walking, linked to plantar hyper-pressure. Where appropriate x-rays and ultrasound assessment were used to excluded other pathology. Patients were enrolled consecutively from the Foot and Ankle Service waiting lists of two consultant surgeons.

\section{Inclusion and Exclusion criteria}

Patients were included who had mechanical metatarsalgia. Patients were included with historical hallux valgus surgery that had persistent or developed new onset metatarsalgia, as were patients having lesser toe straightening and subluxations of the metatarsal phalangeal joint. However patients having concurrent surgery including hallux valgus correction, $1^{\text {st }}$ MTP fusion, or gastrocnemius lengthening were excluded, as were those with a clinical diagnosis of Morton's neuroma, lesser MTP degenerative joints, plantar plate tear, MTP dislocation equinus deformity, cavus foot deformity or inflammatory arthritis. To ensure independence of the data, if a bilateral procedure was undertaken then questionnaire data relating to only one procedure were included; data relating to the procedure with the smallest pre-postoperative MOXFQ score change was used.

\section{Procedures and Outcomes}

All operations were undertaken by one of two specialist foot and ankle consultant surgeons. All patients were examined and the MOXFQ $\left({ }^{\circledR}\right.$ Copyright Oxford University Innovation Limited 2006) outcome measure recorded three weeks pre-operatively and at least 1 year postoperatively. The MOXFQ is a validated scoring system suitable for measuring all foot and ankle surgery [13,14]. The MOXFQ contains 16 items, comprising three dimensions: walking/standing (seven items), foot pain (five items) and social interaction (four items). The three domain scores and the overall index score were converted to 
a metric scale 0 to 100 , where 0 is the best score possible and represents an excellent outcome [13]. In addition patient electronic records were evaluated to establish any operative complications.

A further supplementary question asked patients "How satisfied are you with your operated foot?" This was answered on a five-point Likert scale with options: Very satisfied, Satisfied, Neither satisfied nor dissatisfied, Dissatisfied, Very dissatisfied.

AP weight bearing radiographs were taken in advance of surgery and at least 6 weeks following surgery. Radiographs were accessed and reviewed using the electronic PACs system (Carestream Health Inc., New York, NY). The x-rays were marked using PACS software and following Maestros measurements [7].

\section{Operative Technique}

This procedure may be performed under local anaesthesia, however all of the DMMO procedures in this study were performed under a general anaesthetic. A stab incision is made dorsally, slightly medial or lateral to the metatarsal head with a beaver blade. Distal metatarsal osteotomies are performed using a full cutting straight $2 \times 12 \mathrm{~mm}$ Shannon burr on the distal metaphyseal of the metatarsal. The osteotomy is an extra-articular cut, which is oblique, starting plantar and proximally moving to dorsal and distal, with a $45^{\circ}$ angulation of the metatarsal shaft (see figure 2). The fragments are moved in dorsal plantar direction in the sagittal plane to ensure mobilization of the metatarsal head and that the osteotomy is complete. No fixation was used in these metaphyseal extra-articular osteotomies to enable shortening and elevation of the metatarsal head to decrease the plantar pressure. 


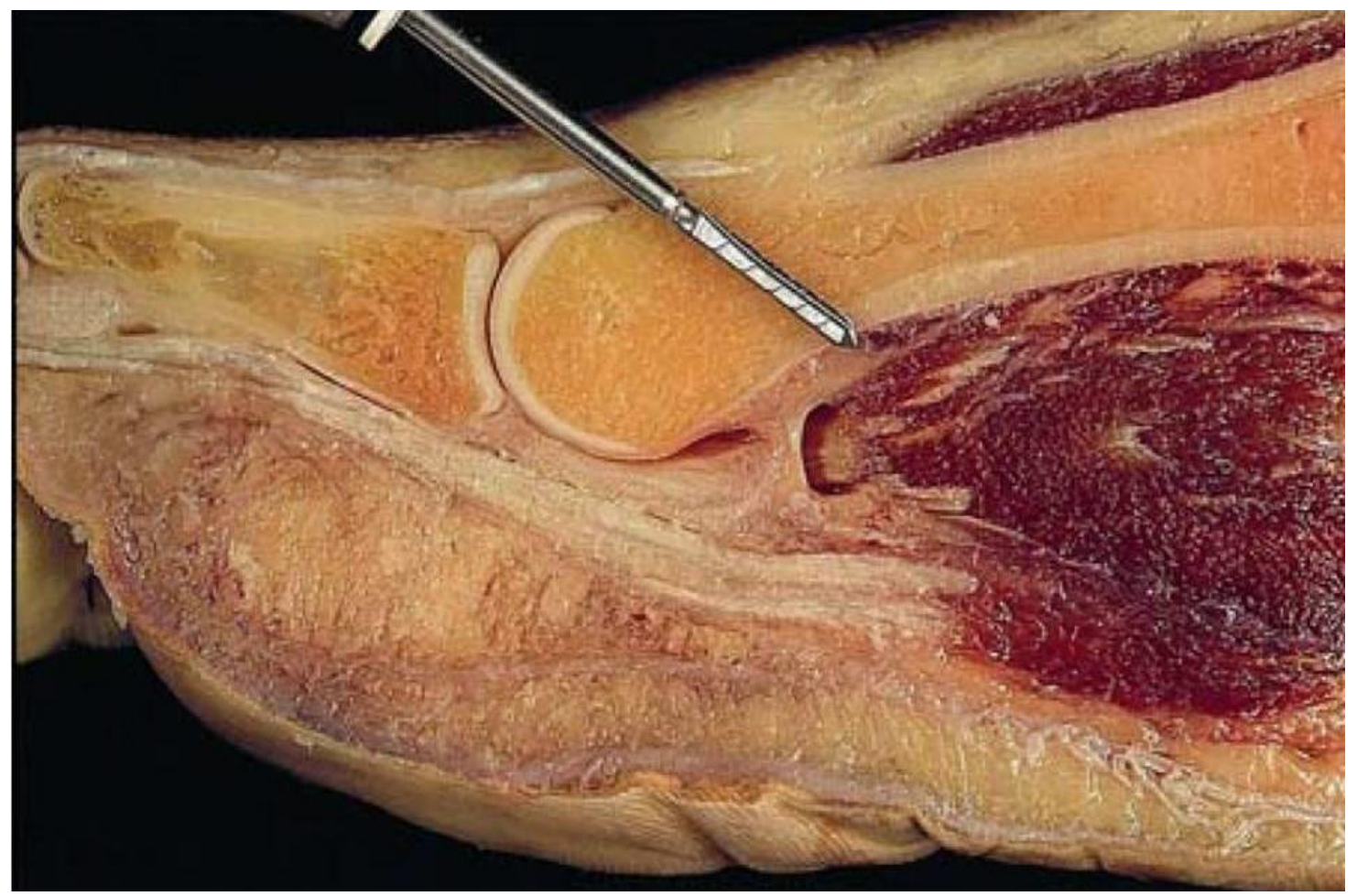

Figure 2. MIS DMMO with a $45^{\circ}$ burr angulation to the metatarsal shaft [6]

Steri-strips were used to close the incision sites. The foot was dressed using sterile wool and crepe bandage. Patients were encouraged to weight bear immediately in a rigid soled surgical shoe as tolerated.

Descriptive and inferential analyses were undertaken using Microsoft Excel (Microsoft Corp., USA) and SPSS statistical software (v24) (IBM Corp, USA). The data were tested for normality using the Shapiro-Wilk test.

\section{Results}

There were 57 patients identified who had the DMMO procedure. 32 patients were excluded, as per the exclusion criteria. This included two patients with inflammatory arthritis, and patients with concurrent surgery including: eleven who had undergone Scarf/Akin procedures, eleven $1^{\text {st }}$ MTP fusion, four with gastrocnemius lengthening, one with a Weils osteotomy, one with Tarsalmetatarsal fusion and one with revision Akin. Two patients had bilateral surgery 
and therefore only one set of pre and postoperative questionnaires was used for each of these patients. One patient died of natural causes (unrelated to her surgery) and was lost to follow up. Hence, there were 24 patients meeting the inclusion/exclusion criteria of whom 20 were women and 4 were men, with a mean age at the time of surgery of 64 years (sd 8.6), BMI 31 (sd 7.5). There were two patients with a diagnosis of osteoporosis and two with diabetes. Three patients had historical scarf Akin procedures and two $1^{\text {st }}$ MTP fusion. Using the Shapiro-Wilk test the preoperative data were normally distributed but the postoperative data were not. Therefore both the mean and median results are presented (Table 1).

Table 1 Pre and postoperative MOXFQ metric scores

\begin{tabular}{lcccccccc}
\multicolumn{3}{c}{ Pre-op score } & \multicolumn{7}{c}{ Post-op score } \\
& Walking & Pain & Social & Index Score & Walking & Pain & Social & Index \\
Median & 55.4 & 55.0 & 43.8 & 54.7 & 12.5 & 25 & 12.5 & 19.5 \\
Range & $0-100$ & $20-100$ & $0-100$ & $9.4-95.3$ & $0-78.6$ & $0-60$ & $0-62.5$ & $0-65$ \\
& & & & & & & & \\
Mean & 57.3 & 56.5 & 44.5 & 53.8 & 23.7 & 25.6 & 24.0 & 24.3 \\
sd & 26.3 & 19.6 & 25.4 & 20.3 & 26.6 & 21.2 & 22.2 & 21.8
\end{tabular}

$\mathrm{N}=24$. ${ }^{*}$ note 0 = best possible score, $100=$ worst possible score

The pre and postoperative mean and median MOXFQ index scores are represented graphically in Figure 3.

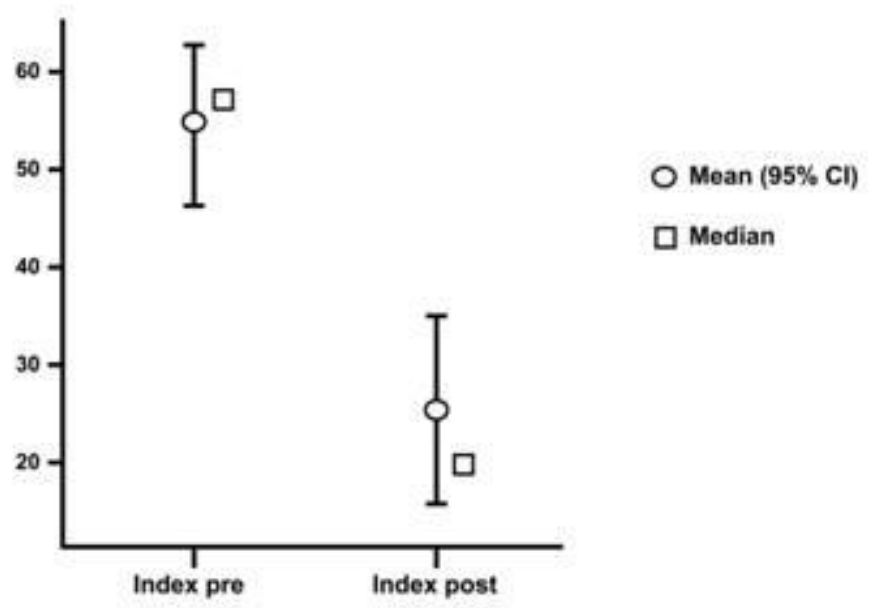


Figure 3. Plot illustrating the mean and median pre and postoperative index scores

Shapiro-Wilk tests indicated the change scores for each domain and the overall index score were normally distributed therefore Paired Samples T-Tests were used to test pre to postoperative mean differences. Statistically significant improvements in pre-post surgery were found for each of the MOXFQ domain scores and the index score (see table 2). A mean difference of 29.5 in pre/postoperative Index score represents clinically significant improvement.

Table 2. Mean differences between MOXFQ scores pre and post surgery

\begin{tabular}{lrrrrrr} 
& \multicolumn{7}{c}{$\begin{array}{c}\text { M5\% Confidence } \\
\text { Interval } \\
\text { difference }\end{array}$} & Lower & Upper & t & df & p \\
\cline { 2 - 7 } & 33.6 & 21.1 & 46.2 & 5.55 & 23.0 & $<.001$ \\
Walking & 30.8 & 20.5 & 41.1 & 6.19 & 23.0 & $<.001$ \\
Pain & 20.6 & 8.7 & 32.5 & 3.57 & 23.0 & 0.002 \\
Social & 29.5 & 19.1 & 39.9 & 5.86 & 23.0 & $<.001$ \\
Index & \multicolumn{7}{c}{} & & & & &
\end{tabular}

Twenty four patients responded to the question "How satisfied are you with your operated foot?" using a 5 point Likert scale. The majority of patients reported excellent satisfaction (see Figure 4). Of the 24 respondents $79 \%(n=19)$ reported a satisfied or very satisfied, $17 \%(n=4)$ indifferent, $4 \%(n=1)$ dissatisfied, $0 \%$ $(\mathrm{n}=0)$ very dissatisfied outcome of surgery. 


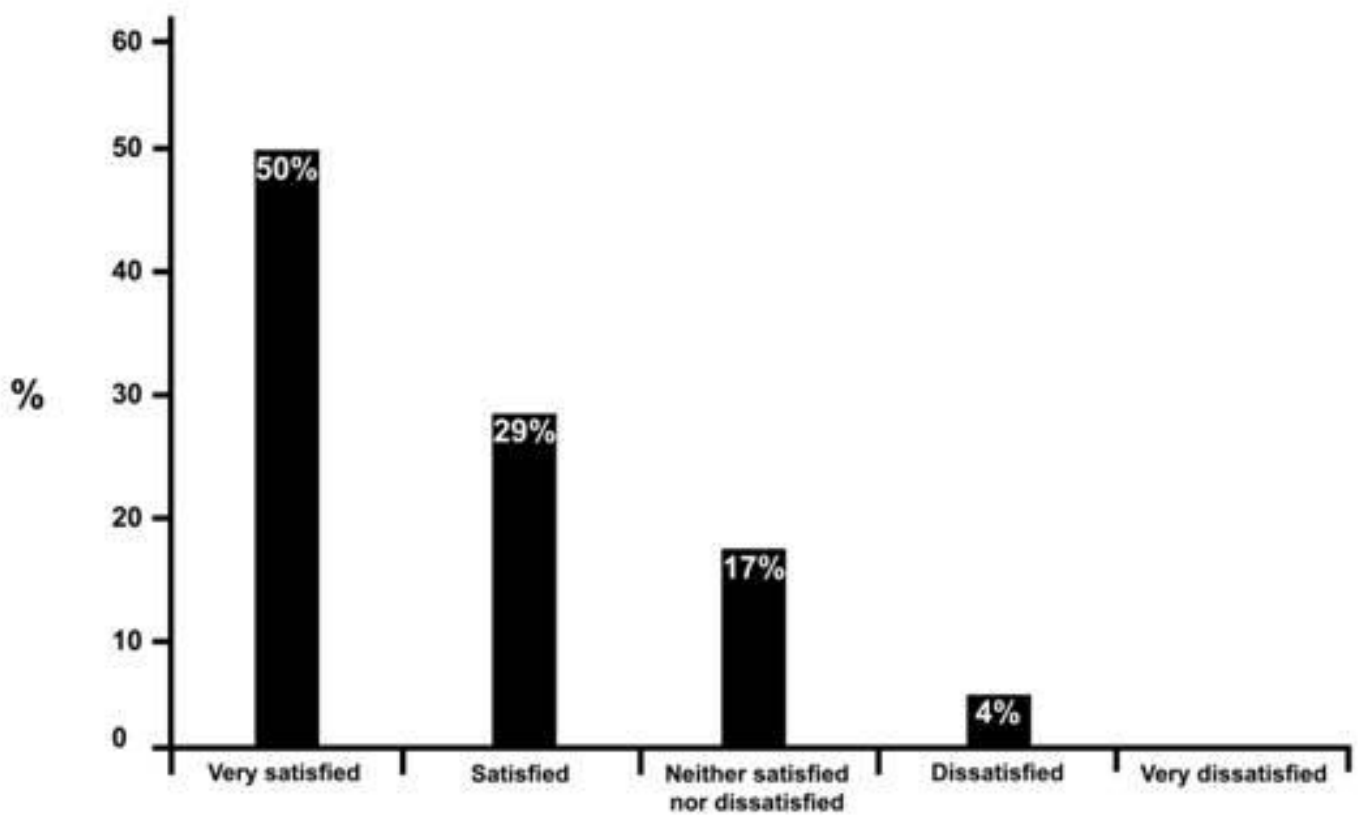

Figure 4. Bar chart demonstrating patient satisfaction following the DMMO procedure

The average recoil of the metatarsal heads following DMMO; M2 4.01mm, M3 $4.55 \mathrm{~mm}, \mathrm{M} 44.16 \mathrm{~mm}$. There were no cases of nonunion and no revision surgery was necessary. There were three recorded complications following the DMMO. One broken burr intra operatively was recovered through the minimal incision site without further incident. One patient suffered both gastro-intestinal bleed following non steroidal anti-inflammatory drugs and pulmonary embolism (VTE). One patient experienced delayed union which demonstrated hypertrophic bone formation (see Figure 5). 


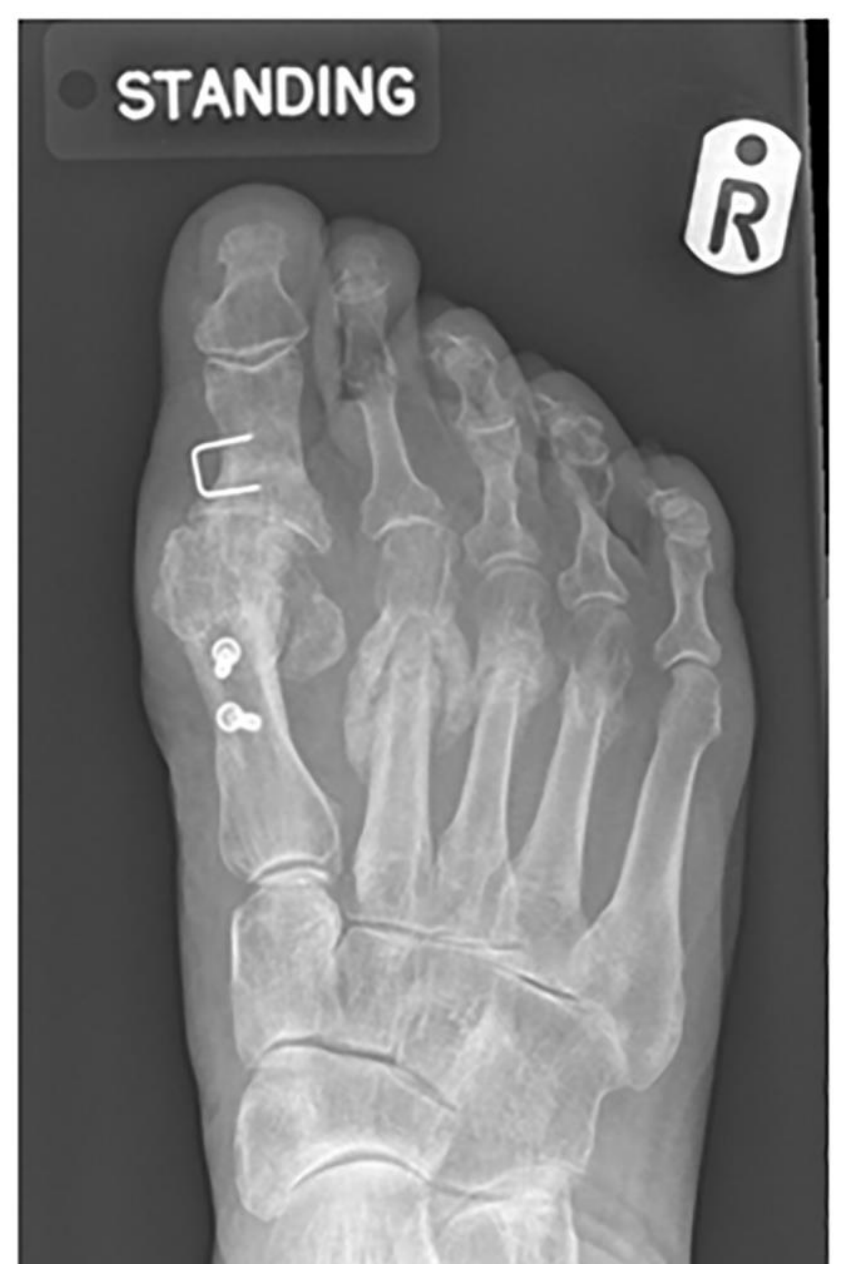

Figure 5. Hypertrophic bone formation following DMMO

\section{Discussion}

This study provides further support that the DMMO offers an alternative to the Weil osteotomy. Previous comparative evidence has demonstrated a similar metatarsophalangeal range of motion following DMMO and Weil osteotomy [15]. However, a longer recovery period is reported following DMMO, due to prolonged oedema and delayed bony union. It is proposed that a Weil osteotomy is indicated with metatarsophalangeal joint dislocation or localised propulsive metatarsalgia and a DMMO indicated where there is a generalised static metatarsalgia with a clinically round plantar forefoot without metatarsal parabola imbalance [15].

The current evidence base for using DMMO over other procedures is limited and based on a small number of studies where patients received concurrent $1^{\text {st }}$ ray 
surgery [1-5]. These studies' limitations variously include retrospective postoperative evidence using the non validated American Orthopaedic Foot and Ankle Score (AOFAS) [16] or the absence of preoperative scores to compare Weil and the DMMO therefore the results should be interpreted with caution. The results from the current DMMO study are from a small series, due to exclusion of first ray procedures, but are relevant due to the limited independent research published on the subject. Overall there was a $29.5(95 \% \mathrm{Cl} 19.1$ to 39.9) point reduction in the mean metric MOXFQ index score from a preoperative score of 53.8 to postoperative score of 24.3. The final MOXFQ postoperative score of this study 24.3 appears favourable compared to those previously reported MOXFQ 31[2]. The results support the rejection of the null hypothesis, as a statistically significant difference was established between pre and 1 year postoperative patient reported outcome scores demonstrating that the DMMO is an effective procedure with a clinically important and statistically significant improvement in MOXFQ mean scores at one year post-op. Statistically significant differences were also found between pre and post MOXFQ metric scores on the three domains of Walking/Standing, Pain, and Social Interaction. A limitation of this study is the use of a convenience sample and a `before and after` study design which lacks a comparison group.

Twenty of the patients in our sample reported improvement in MOXFQ index score. Four patients recorded the lowest score of 0 for MOXFQ postoperatively. Concern has been recorded regarding the lack of direct vision when using MIS surgery, with poor outcomes in the 1980s leading to near complete abandonment of the procedure [6]. However, the introduction of new techniques and development of equipment has resulted in DMMO becoming the procedure of choice in some areas [17]. Furthermore a DMMO cadaveric study of 10 feet (30 metatarsals) with appropriate technique has demonstrated minimal risk of 
neurovascular and tendon injury [18]. There are known complications with DMMO and these include; wound infection, non-union [2] mal-union with ongoing symptoms, floating toes, metatarsal head necrosis and transfer metatarsalgia [12]. In the current study one patient experienced a delayed union. One patient who had a BMI greater than 50 and who had bilateral surgery with a procedure time of less than 30 minutes, experienced a gastric bleed as a result of NSAI and also suffered from a pulmonary embolism (VTE). The risk of developing a Venous Thrombo Embolism (VTE) following forefoot surgery, with immediate weightbearing and no previous history or risk factors, is considered to be very low at 0 to $0.55 \%$ however risk factors should be assessed on an individual basis [19].

The patients' satisfaction reported in this study was $79 \%$ very satisfied or satisfied. This is slightly lower when compared to previously reported work using a four point Likert scale with an $88.6 \%$ satisfaction [3]. However, the majority of patients in Magnan et al.'s [3] study also had $1^{\text {st }}$ ray surgery, a factor which could substantially influence outcomes in other papers [4,5].

The postoperative regime of this study was to allow immediate weightbearing as tolerated following surgery in a rigid soled postoperative surgical shoe. A cadaveric study has suggested that the soft tissue sleeve may offer some stability of the metatarsal head offering sufficient stability of the osteotomy [18] and therefore no fixation is required for this procedure. Standard postoperative care allows immediate weightbearing in a rigid soled postoperative surgical shoe $[2,9,12,20]$. However, specific postoperative strapping for 4 weeks has been recommended by some in order to avoid excessive rotation of the metatarsal head $[6,21]$. The average recoil of the metatarsal heads following DMMO was M2 $4.01 \mathrm{~mm}$, M3 $4.55 \mathrm{~mm}, \mathrm{M} 44.16 \mathrm{~mm}$. As the metatarsal head involves retraction, elevation and rotation on weightbearing, a more accurate method of 
evaluation would be a standing CT scan. However our hospital felt that exposing patients to a standard CT for this procedure would not be appropriate. There were no non-unions recorded in this series.

Historically the use of lesser metatarsal osteotomies with no fixation led to a high rate of non-union and so fell out of favour - this was seen with the Helal procedure where a non- union rate of $15.5 \%$ was reported [22]. The Weil osteotomy with fixation was felt to have a much lower risk and is reported to have a non-union/mal-union rate of $3 \%$ [11]. Concerns that DMMO would lead to a similarly high non-union rate have not been found in our study where we had one delayed union but no non-unions. Other published DMMO literature shows a similarly low non-union rate with one non-union and one mal-union in a series of 30 patients [2]. In their study the non-union was attributed to the osteotomy cut being too distal and perpendicular to the long axis of the metatarsal [2].

\section{Conclusion}

Our study demonstrates clinically and statistically significant improvements in patient reported outcomes following DMMO and $79 \%$ of patients satisfied or very satisfied with this procedure. The average recoil of the metatarsal heads following DMMO was M2 $4.01 \mathrm{~mm}, \mathrm{M} 34.55 \mathrm{~mm}, \mathrm{M} 44.16 \mathrm{~mm}$. There was one delayed union and no non-unions which is comparable to other lesser metatarsal osteotomy procedures. Our study has some methodological limitations and there remains the need for a stronger test of the DMMO procedure such as a randomised controlled trial or cohort comparison study. 


\section{Brief Summary}

Existing studies report DMMO outcome scores in the treatment of metatarsalgia which are limited due to:

1. Previous studies report the DMMO procedure with concurrent $1^{\text {st }}$ ray surgery in the treatment of metatarsalgia

2. The use of non validated outcome measures.

3. Retrospective analysis which lack preoperative scores

This study provides:

1. Data for the use of the DMMO as the principal procedure providing pre and post operative scores, and excludes concurrent $1^{\text {st }}$ ray procedures.

2. Validated prospective pre and post operative MOXFQ data. A statistical and clinically significant difference was found in pre and post operative MOXFQ with high patient satisfaction outcomes.

3. Demonstrates average recoil of the metatarsal heads following DMMO was M2 4.01mm, M3 4.55mm, M4 4.16mm.

This research did not receive any specific grant from funding agencies in the public, commercial, or not-for-profit sectors. 


\section{References}

[1] NEM Yeo, B Loh, JY Chen, AKS Yew, SY Ng, Journal of Orthopaedic Surgery. 24 (2016) 350-353.

[2] S Haque, R Kakwani, C Chadwick, MB Davies, CM Blundell, Foot Ankle Int. 37 (2016) 58-63.

[3] B Magnan, I Bonetti, S Negri, T Maluta, C Dall'Oca, E Samaila, Foot and Ankle Surgery. (2017).

[4] C Biz, M Corradin, K Kanah, W Trepin, M Dalmau-Pastor, A Zornetta, et al., BioMed research international. 2018 (2018).

[5] K Malhotra, N Joji, S Mordecai, B Rudge, The Foot. 38 (2019) 43-49.

[6] M De Prado, M Cuervas-Mons, P Golanó, J Vaquero, Techniques in Foot \& Ankle Surgery. 15 (2016) 12-18.

[7] M Maestro, J Besse, M Ragusa, E Berthonnaud, Foot Ankle Clin. 8 (2003) 695-710.

[8] Barouk P., Foot and ankle clinics. 19 (2014) 407-424.

[9] Lui TH, Foot. 24 (2014) 180-5.

[10] WT DeCarbo, D Dial, Techniques in Foot \& Ankle Surgery. 13 (2014) 191 198.

[11] P Highlander, E VonHerbulis, A Gonzalez, J Britt, J Buchman, Foot \& ankle specialist. 4 (2011) 165-170.

[12] T Wong, S Kong, Journal of Orthopaedics, Trauma and Rehabilitation. 17 (2013) 17-21.

[13] D Morley, C Jenkinson, H Doll, G Lavis, R Sharp, P Cooke, et al., Bone Joint Res. 2 (2013) 66-69.

[14] J Dawson, I Boller, H Doll, G Lavis, R Sharp, P Cooke, et al., J.Bone Joint Surg.Br. 94 (2012) 215-221.

[15] J Henry, J Besse, M Fessy, Orthopaedics \& Traumatology: Surgery \& Research. 97 (2011) S57-S65.

[16] T CODE ., (2017).

[17] J Besse, Orthopaedics \& Traumatology: Surgery \& Research. (2017).

[18] V Dhukaram, AP Chapman, PK Upadhyay, Foot Ankle Int. 33 (2012) 1139-1144.

[19] J Mangwani, N Sheikh, M Cichero, D Williamson, The Foot. 25 (2015) 173-178.

[20] T Bauer, E Gaumetou, S Klouche, P Hardy, N Maffulli, The Journal of Foot and Ankle Surgery. 54 (2015) 373-377.

[21] T Bauer, Orthopaedics \& Traumatology: Surgery \& Research. 100 (2014) S191-S204.

[22] B Helal, M Greiss, J.Bone Joint Surg.Br. 66 (1984) 213-217. 